\title{
Extensor Hallucis Capsularis o Tendón Accesorio del Extensor Hallucis Longus: Estudio anatómico y funcional, frecuencia y mediciones
}

\author{
Extensor Hallucis Capsularis or Accessory Tendon of Extensor Hallucis \\ Longus: Functional anatomy, frequency and measurement
}

\author{
Da. Patricia Palomo López (1), Dr. Juan Carlos Prados Frutos (2) \\ Dr. Ricardo Becerro de Bengoa Vallejo (3), Dra. Marta Elena Losa Iglesias (2)
}

(1) Prof. Colaborador Universidad de Extremadura.

(2) Universidad Rey Juan Carlos.

(3) Universidad Complutense de Madrid

\author{
Correspondencia: \\ Patricia Palomo López \\ Centro Universitario de Plasencia. Avda. Virgen del Puerto. \\ 10600 Plasencia (España)
}

Fecha de recepción: 10 noviembre de 2010

Fecha de aceptación: 22 de febrero de 2011

Los autores declaran no tener ningún tipo de interés económico o comercial.

\section{RESUMEN}

El objeto de éste estudio fue observar detenidamente la anatomía del Extensor Hallucis Longus, Ligamento Capsularis o Tendón Accesorio del músculo Extensor Hallucis Longus; definir su su frecuencia de aparición, pues es un músculo que no está presente en todos los pies y suscita controversia en la bibliografía consultada.

El Ligamento Capsularis es un ligamento del grupo dorsal del pie, que acompaña al músculo Extensor Hallucis Longus siempre medial a él.

Pretendemos demostrar con nuestras disecciones, que es un ligamento más habitual de lo que parece y, dónde está su origen e inserción, así como su función, presentación y ubicación mediante fotografías en pies de cadáver, así como medidas sobre su anchura y características particulares.

Para conseguir nuestro objeto de estudio en éste artículo, se diseccionan 50 primeros dedos de cadáver humano, realizando las pertinentes mediciones.

Palabras clave: Extensor Hallucis Capsularis, estudio, anatomía, frecuencia, mediciones.

\section{ABSTRACT}

The purpose of this study was to look closely at the anatomy of the extensor hallucis longus, ligament or tendon capsularis Accessory Extensor hallucis longus muscle; define its occurrence frequency, it is a muscle that is not present in all feet and raises controversy in the reviewed literature. The ligament is a ligament capsularis dorsalis pedis group that accompanies the extensor hallucis longus muscle medial to it forever. We intend to demonstrate by our dissections, a ligament more common than it seems and where is its origin and insertion as well as their function, presentation and location by using photographs of cadaver feet and its width measures and characteristics. To achieve our object of study in this article, first 50 dissected human cadaver fingers, making the relevant measurements.

Key words: Extensor hallucis capsularis, study, anatomy, frequency measurements.

Sumario: 1. Justificación. 2. Objetivos. 3. Desarrollo. 4. Material y método. 5. Resultados. 6. Discusión. 7. Conclusiones. Bibliografía

Referencia bibliográfica: Palomo López P, Prados Frutos JC, Becerro de Bengoa Vallejo R, Losa Iglesias M. Extensor Hallucis Capsularis o Tendón Accesorio del Extensor Hallucis Longus: Estudio anatómico y funcional, frecuencia y mediciones. Rev. Int. Cienc. Podol. 2011; 5(2): 25-34. 


\section{JUSTIFICACIÓN}

La miología permite establecer la existencia de un número determinado de músculos, en éste caso en el pie.

El presente trabajo está referido al estudio de la presencia del Extensor Hallucis Capsularis (EHC), llamado también ligamento capsularis o tendón accesorio del Músculo Extensor Hallucis Longus (EHL), el cual no está siempre presente según los estudios anteriormente realizados.

Los estudios y prácticas realizados en medios académicos, especialmente europeos y norteamericanos, conocidos a través de la literatura especializada en anatomía humana en general y el estudio de los músculos en particular, permite afirmar el carácter no presente siempre de este ligamento, sin embargo, gracias al avance del trabajo científico y observaciones realizadas en nuestro medio, podremos conocer mejor éste ligamento, sus medidas, su función, frecuencia... Es decir, profundizar en el conocimiento de éste EHC, por tanto se justifica la realización de la investigación como un aporte al mejor y mayor conocimiento de la anatomía humana en medios y lugares diferentes.

\section{OBJETIVOS}

General:

- Determinar la frecuencia del ligamento capsularis en nuestro medio.

Específicos:

- Registro bibliográfico actual de la frecuencia del ligamento Capsularis.

- Disección de piezas anatómicas en el Departamento de Anatomía y Embriología Humanas. Facultad de Medicina.UCM.

- Análisis y comparación de la frecuencia en piezas anatómicas.

\section{DESARROLLO}

Empezaremos con una breve descripción del Músculo al que acompaña cuando está presente: el Extensor Hallucis Longus o Extensor propio del primer dedo.

El músculo Extensor Hallucis Longus pertenece al grupo de músculos Regio Cruralis Anterior, es decir, región anterior de la pierna, que comprende cuatro músculos yuxtapuestos de medial a lateral en el orden siguiente: tibial

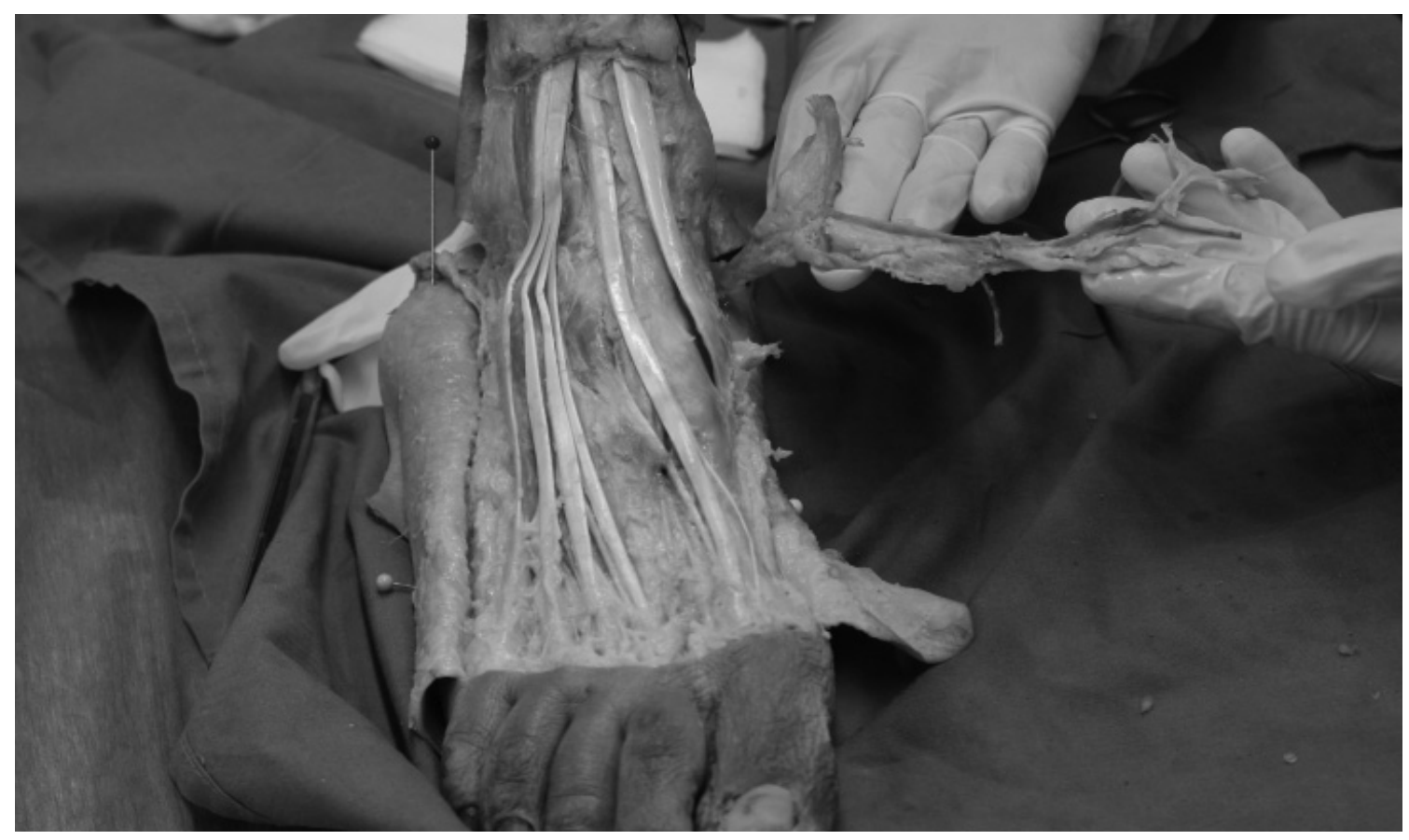

Figura 1. Detalle de la musculatura extensora a nivel del dorso del pie. 
anterior (TA), extensor hallucis longus (EHL), extensor largo de los dedos (ELCD) y peroneo tertius (PT).

\section{Origen}

Fibras carnosas en los dos cuartos medios de la cara cara medial del peroné colocada por delante de la cresta interósea; porción adyacente de la membrana interósea de la pierna y fascia de la pierna, en la parte vecina de dicha membrana $^{5,6,7,8,9,10,11,12,13,14,15,16}$.

\section{Inserción}

La mayoría de autores simplemente describen su inserción en la cara dorsal, a nivel proximal, de la base de la falange distal del dedo gordo $5,8,9,10,11,12,13,14$.

\section{Inervación}

Nervio peroneo profundo (L5-S1) al igual que el extensor digitorum longus y peroneo tertius $^{10,15}$.

\section{Acción}

La mayoría de autores afirman y coinciden en que realiza dorsiflexión del primer dedo y dorsiflexión del tobillo ${ }^{5,8,9,10,11,12} 14$.

\section{Vascularización}

Arteria tibial anterior, hasta el borde inferior del retinaculo inferior de los extensores, donde se convierte en la arteria dorsal del pie (Moore).

La arteria dorsal del pie, desciende anteromedialmente, hasta el primer espacio interóseo del metatarso, el cual atraviesa verticalmente para anastomosarse con la arteria plantar lateral. Esta arteria avanza lateral a lo largo del borde lateral del tendor extensor hallucis longus descansando sobre los huesos del tarso y sus articulaciones.

\section{Tendon accesorio del EHL o Extensor Ha- llucis Capsularis}

Una vez terminada la introducción del EHL, no hay que olvidar que a menudo puede derivarse el tendón accesorio del músculo extensor del dedo gordo, que se inserta en el primer metatarsiano o en el área de la articulación metatarsofalángica, y que se encuentra principalmente en la cara medial del dedo gordo ${ }^{11,1}$; objeto de nuestro estudio.Denominado Extensor Hallucis Capsularis (EHL) ó Ligamento Capsularis.

El extensor hallucis capsularis, es el nombre más común dado al tendón accesorio del EHL, esporádicamente visto medial al EHL.

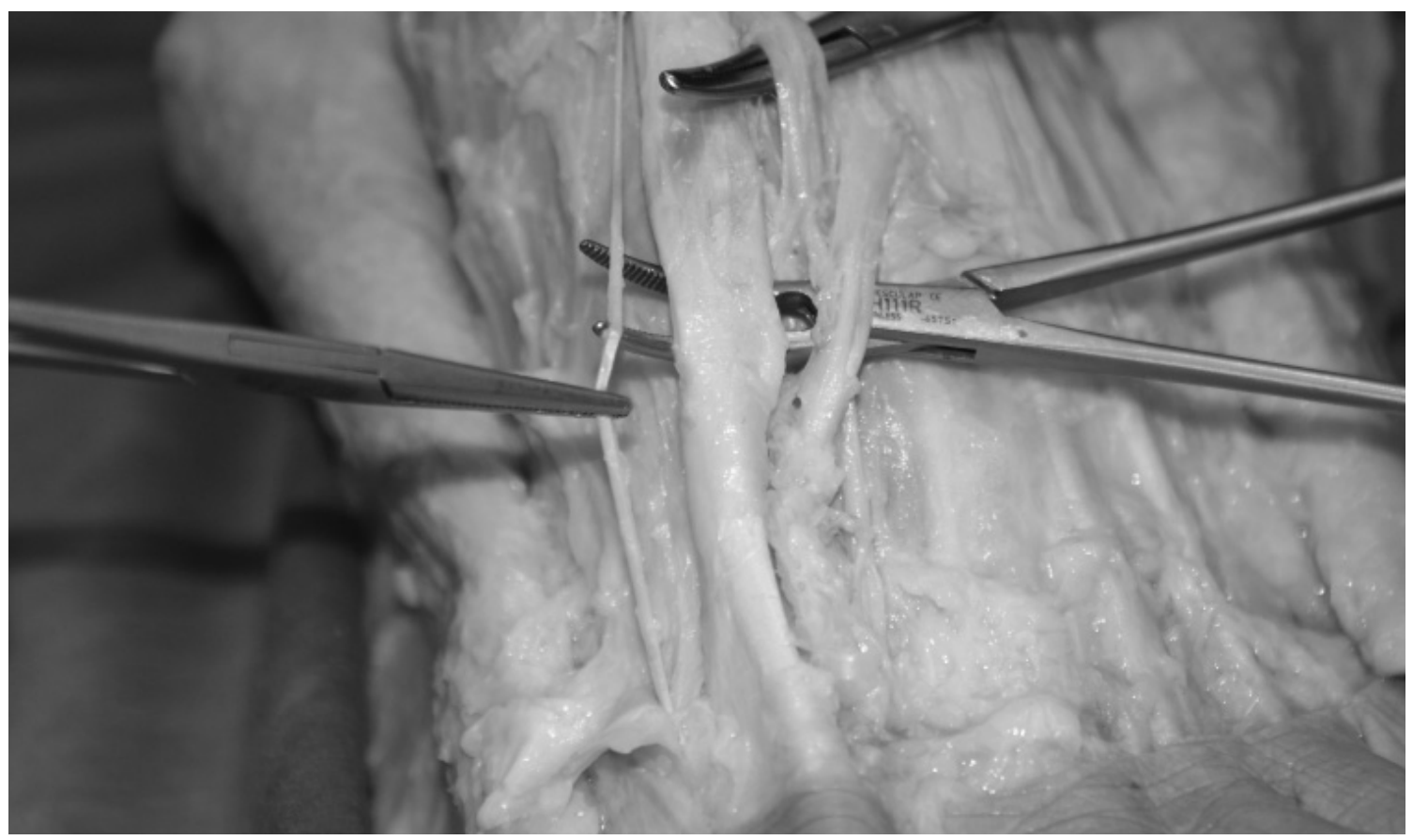

Figura 2. EHC en espécimen 17. 
Entre los pocos estudios encontrados sobre éste músculo accesorio, diremos que tiene su origen en el tendón del EHL en el 93\% de los casos y se inserta en la cápsula de la primera AMTF en el 99\% de los casos ${ }^{4}$; para Bibbo y colaboradores, sus estadísticas para el origen de éste músculo varían poquísimo, en el 92\% de los casos proceden del tendón del EHL y el $8 \%$ restante del tendón del Tibial Anterior (TA); y coincide por completo en la inserción: en la cápsula dorso medial de la primera AMTF.

Según Boyd, el capsularis está presente en el $88 \%$ de los especimenes estudiados, para Bibbo, en el 81\%.

Suelen ser bilaterales en el $80 \%$ de los casos estudiados, y no existen diferencias estadisticas significativas en cuanto a sexo.

Todos los capsularis estudiados presentaban una anchura menor a $2 \mathrm{~mm}$ de ancho ${ }^{4}$, excepto un $16 \%$ que sobrepasaban en poco éstos $2 \mathrm{~mm}$.

Las conclusiones de todos los autores es que éste tendón es único para el pie humano (falta en primates) y es una estructura bastante contante, $80 \%$. Hasta un $14 \%$ de la población pre- sente un tendón capsularis, y además, apto para transplantar.

En la actualidad, la función de éste tendón accesorio en la biomecánica de la primera AMTF sigue siendo bastante desconocida.

El único estudio encontrado en la bibliografía, y al parecer el primero, realizado sobre la frecuencia del EHC en fetos ${ }^{1}$ ), nos dice que éste tendón apareció en el $51 \%$ de los fetos, y dentro de éstos, bilateral en el $65 \%$ y unilateral en el 35\% de los fetos estudiados. Sin estadísticas significativas entre varones y mujeres.

También nos describe como aparece siempre en el lado medial del tendón EHL para insertarse en el borde latero medial de la cápsula articular de la primera AMTF, en todos los especimenes. Es decir, totalmente compatible con los estudios de Boyd, que comentaba que el origen podía ser en el tendón o en el vientre muscular en adultos.

Como hemos estudiado en la literatura, la presencia de EHC es menor en fetos que en adultos, y plantean todos los autores una razón: el uso de EHL en útero materno es práctica-

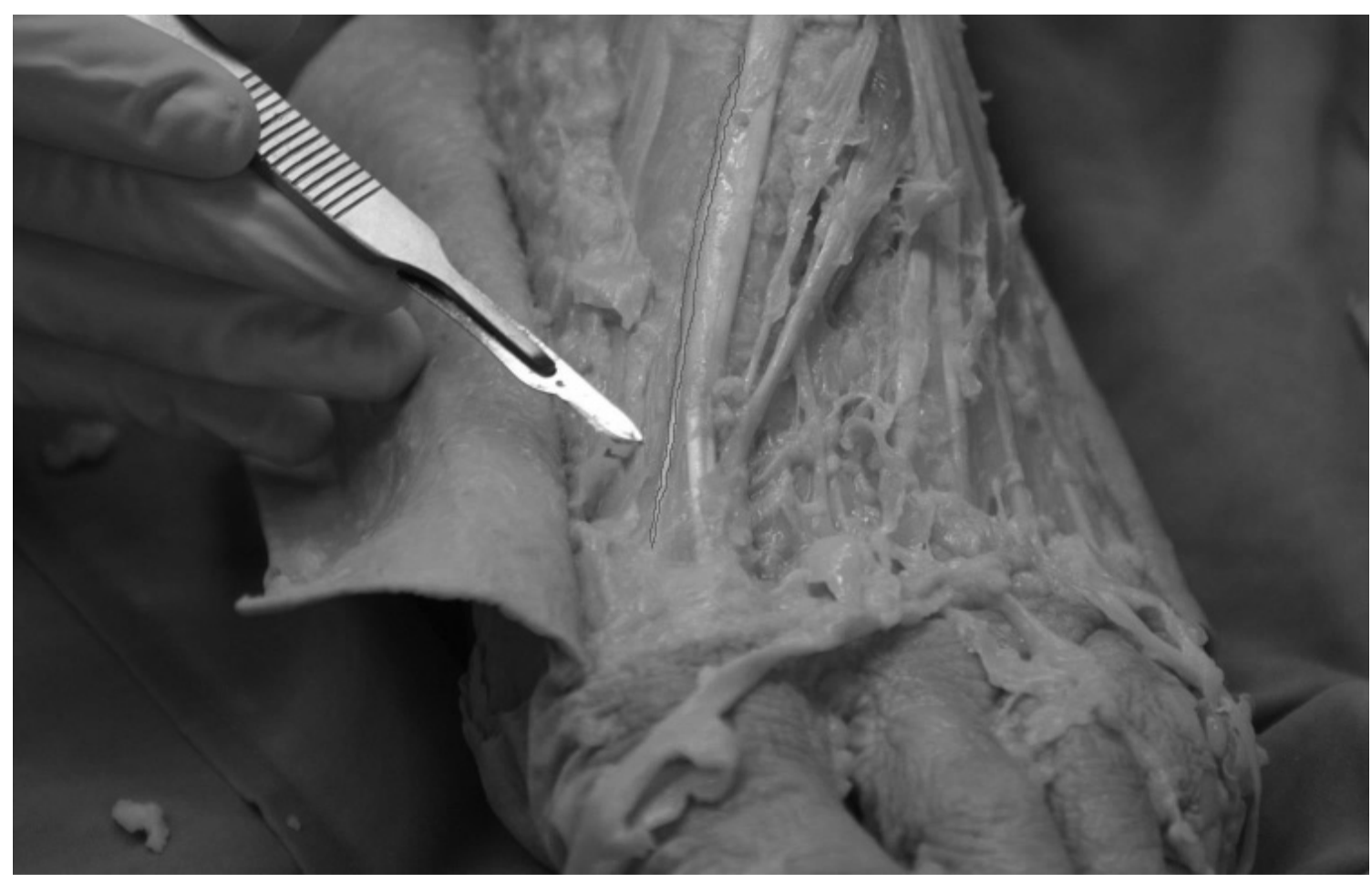

Figura 3. detalle de un EHC en pie adulto, señalado con línea. 
mente nula, lo cual refleja la escasa función del capsularis en fetos. También todos están de acuerdo al que el desarrollo del capsularis es más frecuente después del nacimiento.

Tate et Al (citado en Bibbo et al, 2004 y Boyd et al, 2006), describió la función de éste músculo accesorio como estabilización de la cápsula articular de la primera AMTF cuando existe dorsi-flexión del hallux. No obstante, el resto de autores ${ }^{1,2,3,4}$ plantean la pregunta de si la función del EHC puede ser tras la fijación del tendón principal a la falange proximal y/o la contribución a la extensión de la falange proximal, principalmente, y no sólo la función de estabilización de la cápsula articular.

Se considera que la presencia de capsularis, puede ser uno de los cambios evolutivos del ser humano para adaptarse a la posición bípeda, a la marcha humana, para aliviar la carga y la deambulación.

Vamos a definir la relación entre tendón extensor y la frecuencia del ligamento capsularis acompañando al tendón extensor.

\section{MATERIAL Y MÉTODO}

1. 50 pies de cadáveres humanos frescos se han utilizado para éste estudio, fijados en solución con un componente de formol. En total, registrados 50 primeros dedos de pies, 50 disecciones realizadas.

- Todos ellos pies sin evidencias de trauma, no afectos de patologías deformantes.

- Pies sin intervenciones quirúrgicas de miembros inferiores, ni patologías/ cirugías vasculares.

- 30 pies derechos y 20 pies izquierdos. Todos ellos pies de adultos, ningún niño. Hombres y mujeres por igual.

- Rango de edad entre 45 y 80 años.

2. 6 pies de fetos de cadáver humano, congelados. Sus 6 primeros dedos. 6 disecciones realizadas.

- Una hembra y dos varones, sin patologías ni alteraciones deformantes.

- Todas las medidas se han realizado con fotografías mediante cámara reflex-

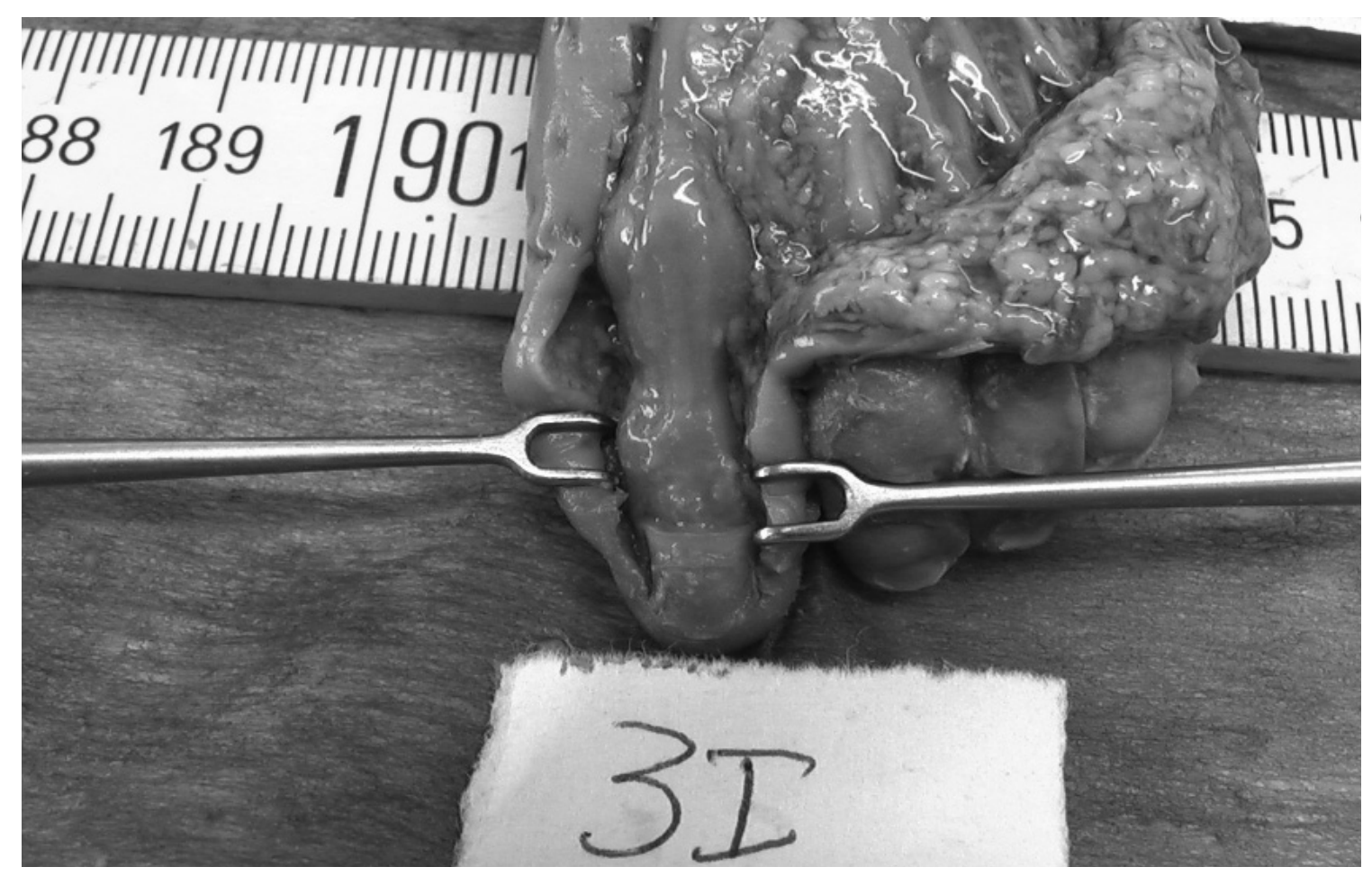

Figura 4. disección de feto. No se aprecia capsularis. 
digital Sony alfa 200, y colocando en todas las fotografías un calibre de precisión y un metro amarillo de carpintero, para poder tomar referencias.

- Se amplian las fotos a una escala de 1:4 y se miden con el sofware autocad.

\section{En los primeros dedos}

1. Disección en la región anterior del pie. Los límites de la garganta del pie: a nivel proximal, por un plano tranverso que pasa por la base de los maléolos y por abajo, otro plano transverso a $3 \mathrm{cms}$ por encima de la línea tarso metatarsiana. Laterales, dos líneas verticales que siguen respectivamente el borde interno y externo de la garganta del pie.

2. La posición del cadáver: pie en decubito supino, extendido fuertemente para mostrar el aparato extensor.

3. Incisión de los tegumentos: se trazan con un rotulador, dos líneas horizontales en los límites superior e inferior de la región, uni- das por una línea vertical media que pase exactamente por el eje de la garganta del pie. Encima de éstas líneas incidimos con el bisturí (nunca de manera oblicua), y cogiéndolo de manera cómoda de forma que seamos nosotros los que controlamos el bisturí. Así, quedan definidos dos colgajos que dispuestos hacia afuera nos descubren el borde anterior de los dos maléolos. Hemos alargado ésta incisión longitudinal hasta la zona metatarsofalangica y, una vez aquí, realizamos dos incisiones perpendiculares hacia lateral y medial. El cadáver del pie, una vez disecados los colgajos, nos quedaría así:

4. A la vez que incidimos en piel, lo hemos hecho también en tejido celular subcutáneo hasta llegar hasta la aponeurosis superficial. Lo primero en identificar es la vena safena interna, voluminosa, y ramas nerviosa que provienen en su mayoría del nervio músculo cutáneo.

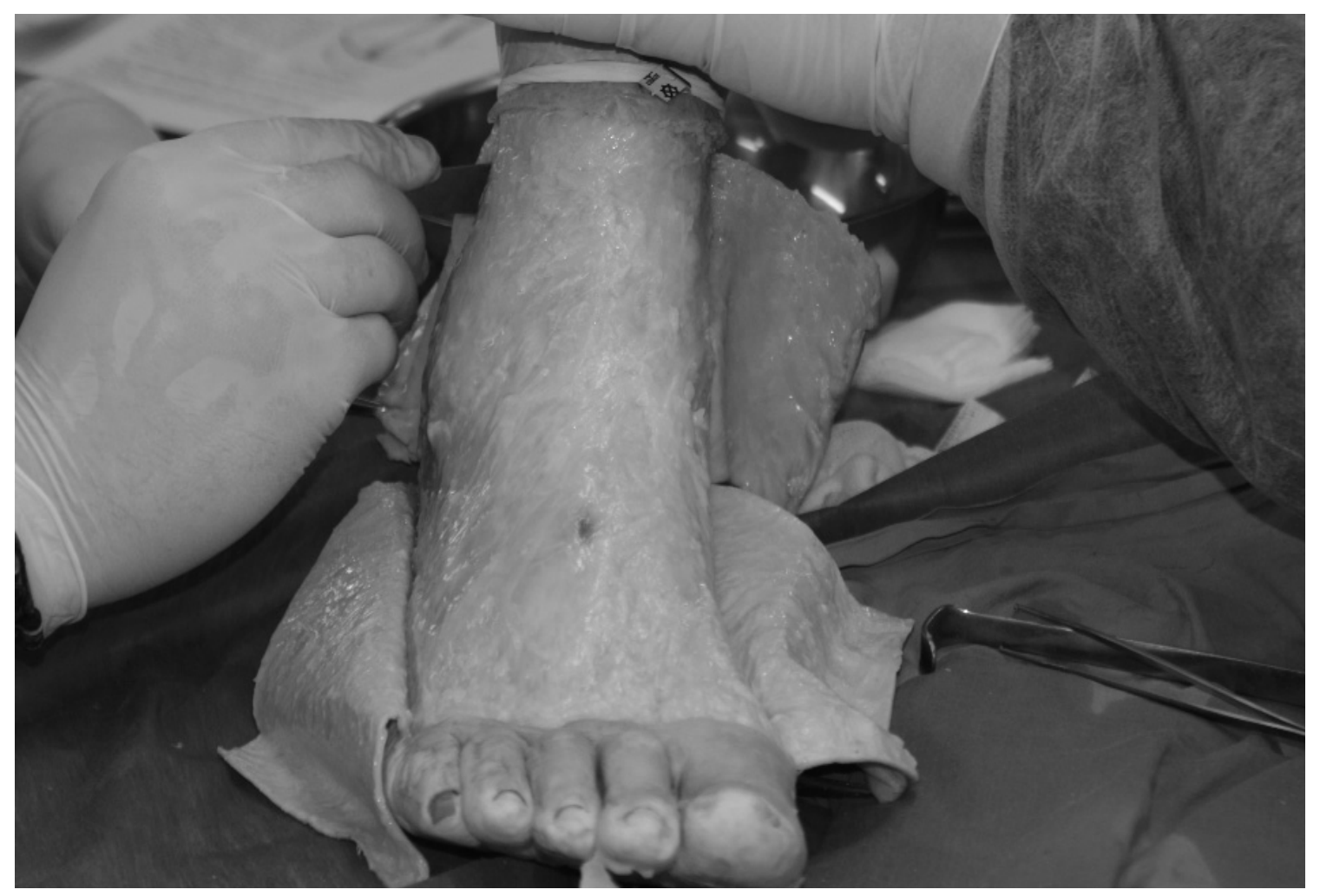

Figura 5. Disección dorsal de la garganta del pie. 
La porción reforzada de la aponeurosis y que cubre a mayor parte de la garganta anterior del pie es el ligamento anular, en forma de y acostada. Levantar éste ligamento en una pieza única no es fácil.

Después llegamos a la capa subaponeurótica. Seguimos disecando y desde dentro hacia afuera, de medial a lateral, distinguiremos:

- el músculo tibial anterior: su tendón, el más interno de los tendones de la cara dorsal del pie, es fácil de reconocer por $\mathrm{su}$ volumen y superioridad sobre los demás. Va acompañado en casi toda la travesía de la zona por su vaina sinovial, que se aprecia muy bien.

- Extensor propio del dedo gordo: también se evidencia una vaina en su trayecto.

- Ligamento Capsularis cuando está pre$\underline{\text { sente }}$

- Vasos y nervios tibiales anteriores.
- Extensor común de los dedos y peroneo anterior.

5. Se comprueba la existencia del tendón accesorio hallucis longus o ligamento capsularis, o extensor hallucis capsularis

6. Se mide la anchura del extensor hallucis capsularis.

La medida de cada una de éstas estructuras es definida en la sección de resultados. Las medidas para cada dedo fueron registradas separadamente y comparadas, se obtienen los rangos.

\section{En Fetos, los primeros dedos}

El método se realiza de la misma manera que para pies adultos.

1. Se mide la anchura del tendón extensor a nivel de la interlínea articular, primera AMTF.

2. Se comprueba la existencia del tendon accesory hallucis longus muscle o ligamento capsularis.

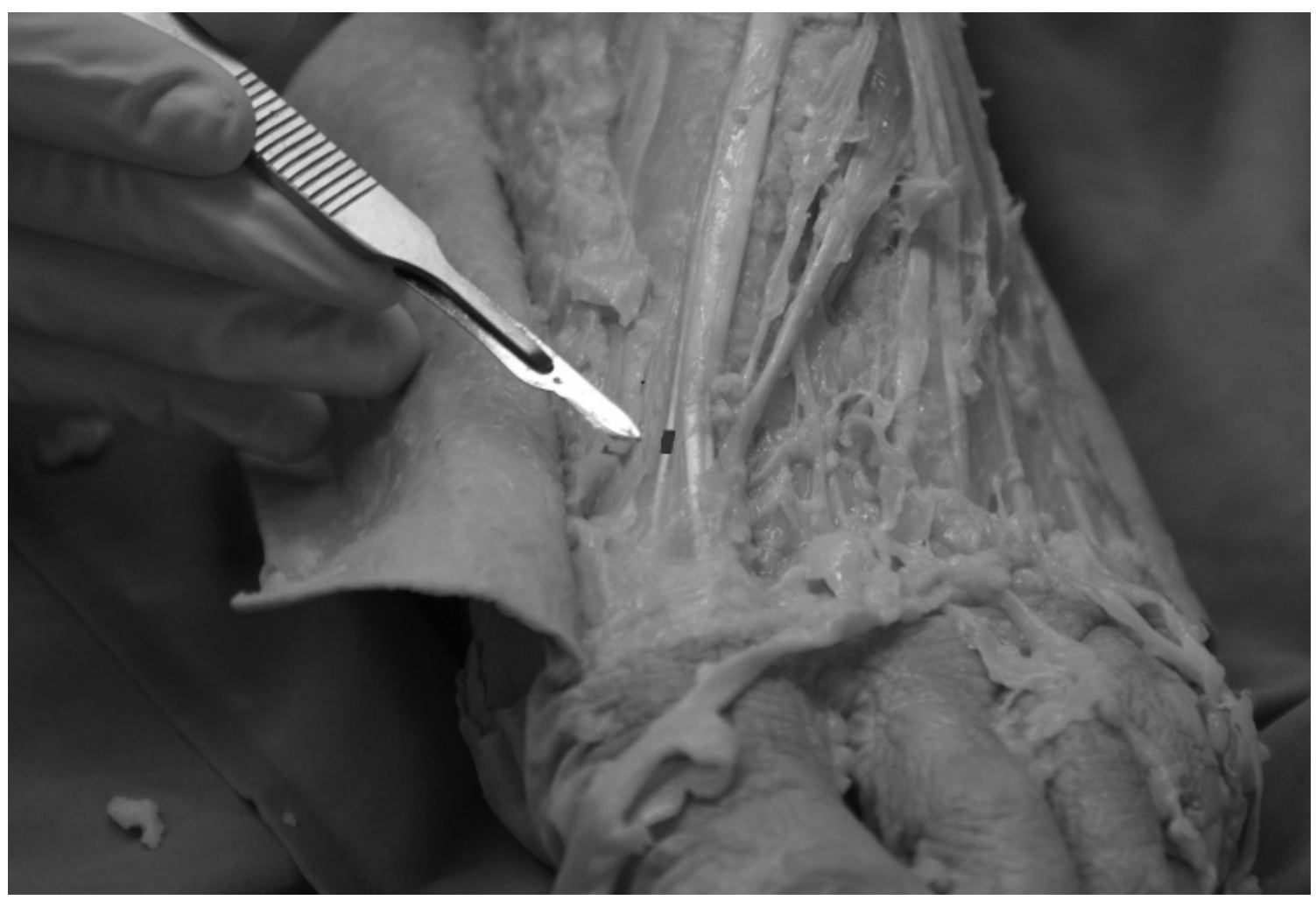

Figura 6. Medición de la anchura del EH capsularis, señalado con línea. 


\section{RESULTADOS}

El tendón extensor del Músculo Extensor Hallucis Longus y Ligamento Capsularis fue inspeccionado en todos los especímenes.

Resultados de las Mediciones con Auto-Cad y Adobe Photoshop en las estructuras anatómicas estudiadas:

\section{DISCUSIÓN}

Según los resultados de esta investigación, hemos comprobado en los especimenes estudiados:
El tendón es la estructura fundamental, responsable de la extensión o flexión dorsal de la articulación interfalángica proximal, y que, la más problable función de las estructuras adyacentes, es proporcionar estabilidad al propio tendón, compartiendo afirmación con los estudios realizados en los dedos de la mano. (Schweitzer 1.)

La anchura del tendón del EHL, según nuestro estudio, nos ofrece un rango menor de 3.4 y mayor de $5.5 \mathrm{~mm}$ con una desviación estándar de $4.4 \mathrm{~mm}$; y el grosor, presenta un rango menor de $1 \mathrm{~mm}$ y rango mayor de 2.6 $\mathrm{mm}$, con una desviación estándar de $1.49 \mathrm{~mm}$, es decir, de naturaleza fina.

\begin{tabular}{|l|l|l|}
\hline Fetos: & $\begin{array}{l}\text { Anchura tendón a la altura } \\
\text { interlínea articular }\end{array}$ & $\begin{array}{l}\text { Presencia accesorio } \\
\text { capsularis }\end{array}$ \\
\hline 1 Dcho: F & 1.8 & No \\
\hline 1 Izdo : F & 1.9 & No \\
\hline 2 Dcho:M & 2.1 & No \\
\hline 2 Izdo :M & 2.6 & No \\
\hline 3 Dcho :M \\
MM & 2.43 & No \\
\hline 3 Izdo : M & 2.86 & No \\
\hline desviación & 2.27 & \\
\hline
\end{tabular}

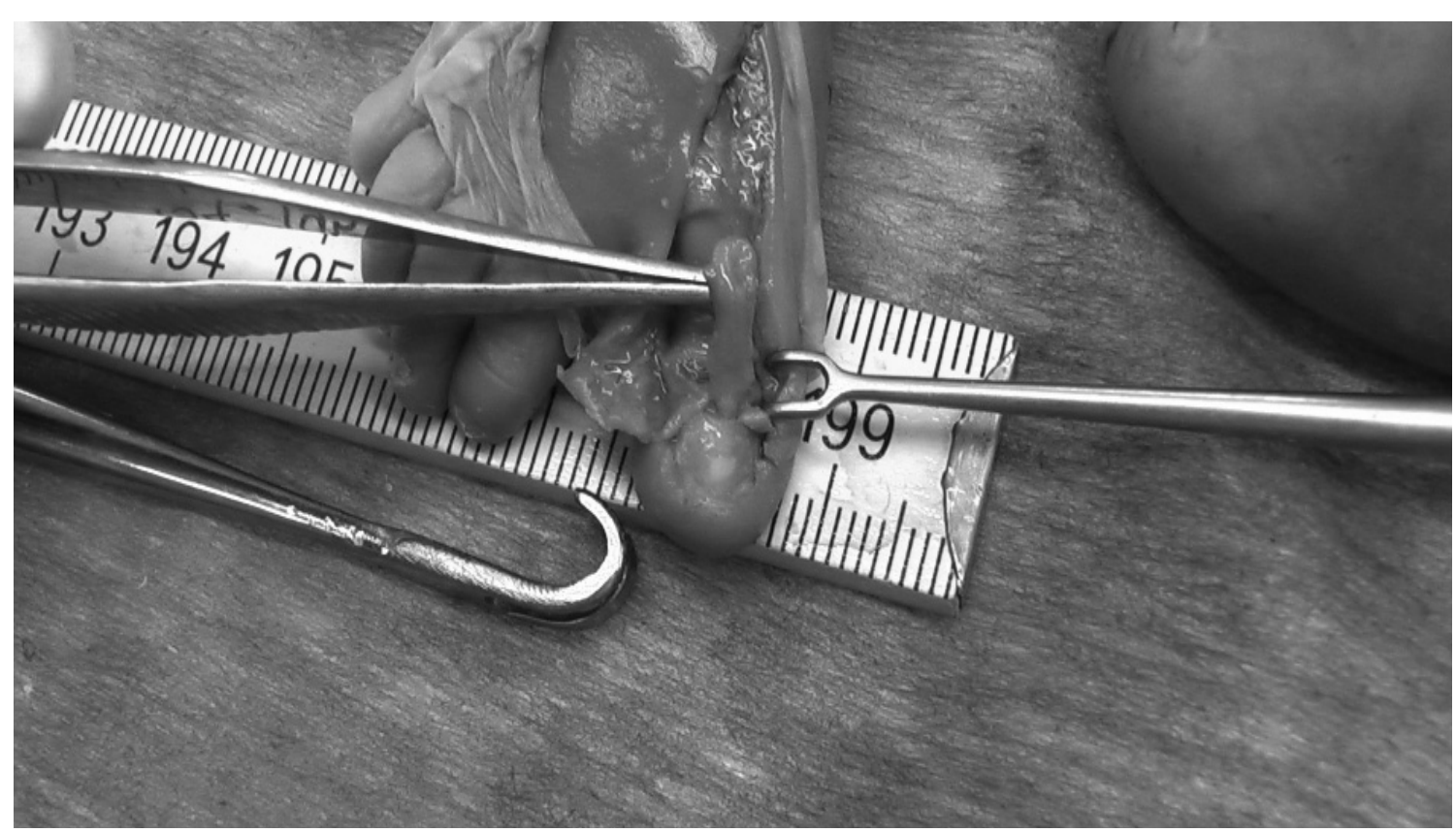

Figura 7. Tendón del EHL en feto dos para proceder a su medición. 


\begin{tabular}{|c|c|c|c|c|}
\hline $\begin{array}{l}\text { Estructuras Anatómicas } \\
\text { Medidas }(\mathbf{m m}) \text { en primeros dedos }\end{array}$ & $\begin{array}{l}\text { Anchura Tendón } \\
\text { EHL }\end{array}$ & $\begin{array}{l}\text { Grueso } \\
\text { Tendón } \\
\text { EHC }\end{array}$ & $\begin{array}{l}\text { Presencia Lig. } \\
\text { Capsularis }\end{array}$ & $\begin{array}{l}\text { Anchura Lig } \\
\text { Capsularis }\end{array}$ \\
\hline Pie 1 & 5.1 & 1 & Sí & 0.99 \\
\hline Pie 2 & 5.1 & 1.5 & No & \\
\hline Pie 3 & 3.6 & 1 & Sí & 1.2 \\
\hline Pie 4 & 5.5 & 1.5 & Sí & 1.13 \\
\hline Pie 5 & 3.8 & 2.6 & Sí & 1.27 \\
\hline Pie 6 & 4.9 & 1.1 & No & \\
\hline Pie 7 & 4.3 & 1.8 & Sí & 1 \\
\hline Pie 8 & 3.8 & 1.6 & Sí & 1.21 \\
\hline Pie 9 & 3.4 & 1.6 & Sí & 0.95 \\
\hline Pie 10 & 5.1 & 1.2 & Sí & 1.41 \\
\hline Pie 11 & 4.8 & 1.4 & No & \\
\hline Pie 12 & 4.4 & 1.7 & Sí & 1.44 \\
\hline Pie 13 & 5.1 & 1 & Sí & 1.28 \\
\hline Pie 14 & 3.8 & 1 & Sí & 1.45 \\
\hline Pie 15 & 5.1 & 1.7 & Sí & 1.3 \\
\hline Pie 16 & 3.6 & 1.4 & Sí & 1.41 \\
\hline Pie 17 & 3.8 & 1.3 & Sí & 1.23 \\
\hline Pie 18 & 5.1 & 1.6 & No & \\
\hline Pie 19 & 3.9 & 1.6 & Sí & 1.26 \\
\hline Pie 20 & 5.4 & 2.5 & Sí & 0.98 \\
\hline Pie 21 & 5 & 1 & Sí & 0.96 \\
\hline Pie 22 & 5 & 1 & Sí & 1.31 \\
\hline Pie 23 & 3.6 & 1.6 & Sí & 1.33 \\
\hline Pie 24 & 3.8 & 1.4 & Sí & 1.15 \\
\hline Pie 25 & 3.7 & 1.7 & No & \\
\hline Pie 26 & 3.7 & 1.7 & Sí & 1.17 \\
\hline Pie 27 & 5.3 & 1.4 & Sí & 1.3 \\
\hline Pie 28 & 5.1 & 1 & No & \\
\hline Pie 29 & 4.5 & 1 & Sí & 1.3 \\
\hline Pie 30 & 4.9 & 2.3 & Sí & 1.37 \\
\hline Pie 31 & 4.9 & 1.4 & Sí & 1.24 \\
\hline Pie 32 & 4.8 & 1.4 & Sí & 1.27 \\
\hline Pie 33 & 3.9 & 1.4 & Sí & 1.35 \\
\hline Pie 34 & 3.8 & 1.7 & Sí & 1.38 \\
\hline Pie 35 & 3.9 & 1.7 & No & \\
\hline Pie 36 & 3.5 & 1.5 & Sí & 1.4 \\
\hline Pie 37 & 5.2 & 1.2 & Sí & 1.48 \\
\hline Pie 38 & 5.2 & 1.6 & Sí & 1.27 \\
\hline Pie 39 & 4.6 & 1.6 & Sí & 1.22 \\
\hline Pie 40 & 4.8 & 2.5 & No & \\
\hline Pie 41 & 5.3 & 1 & Sí & 1.33 \\
\hline Pie 42 & 5.3 & 1 & No & \\
\hline Pie 43 & 5.2 & 1.4 & Sí & 1.4 \\
\hline Pie 44 & 3.8 & 1.4 & Sí & 1.41 \\
\hline Pie 45 & 3.8 & 2 & No & \\
\hline Pie 46 & 4.6 & 1.7 & Sí & 1.23 \\
\hline Pie 47 & 3.6 & 1.7 & Sí & 1.26 \\
\hline Pie 48 & 4.6 & 1.6 & Sí & 0.98 \\
\hline Pie 49 & 3.6 & 1.5 & Sí & 0.99 \\
\hline Pie 50 & 3.7 & 1.3 & No & \\
\hline $\begin{array}{l}\text { Desviación estándar } \\
\text { Rangos menor y mayor }\end{array}$ & $\begin{array}{l}4.4 \\
(3.4-5.5)\end{array}$ & $\begin{array}{l}1.49 \\
(1-2.6)\end{array}$ & Sí 39/50 & $\begin{array}{l}1.24 \\
(0.95-1.45)\end{array}$ \\
\hline
\end{tabular}


Respecto a la frecuencia del ligamento capsularis (EHC) acompañando al tendón del extensor, en el caso de los fetos, ha sido nula, pues no hemos encontrado EHC en ninguno de los especimenes diseccionados. Defenderíamos la teoría compartida con otros autores sobre éste ligamento: el uso de EHL en útero materno es prácticamente nula, lo cual refleja la escasa función del capsularis en fetos, y por tanto, su baja frecuencia en fetos. También todos están de acuerdo al que el desarrollo del capsularis es más frecuente después del nacimiento, hasta el punto, de que hemos encontrado EHC en adultos, en el $78 \%$ de los especimenes estudiados, muy similar a los estudios de Bibbo con un $80 \%$ y Boyd con un $88 \%$; y todos con la misma inserción: borde latero-medial de la cápsula articular de la primera AMTF (platzer, aktekin). $\mathrm{Su}$ anchura es nuestro estudio es menor de $2 \mathrm{~mm}$ siempre, con una media de $1.2 \mathrm{~mm}$.

La función de éste músculo accesorio para nosotros sería la estabilización de la cápsula articular de la primera AMTF cuando existe dorsi-flexión del hallux. No obstante, el resto de autores (Aktekin, Boyd,Bibbo,Al-Saggaf), plantean la pregunta de si la función del EHC puede ser tras la fijación del tendón principal a la falange proximal y/o la contribución a la extensión de la falange proximal, principalmente, y no sólo la función de estabilización de la cápsula articular.

Consideramos que la presencia de capsularis, puede ser uno de los cambios evolutivos del ser humano para adaptarse a la posición bípeda, a la marcha humana, para aliviar la carga y la deambulación.

\section{CONCLUSIONES}

Que la presencia del ligamento Extensor Hallucis Capsularis es bastante frecuente, ya que aparece en el 78\% de los especimenes estudiados, excepto en fetos, que no suele existir. Que su anchura tiene una desviación estándar de $1,24 \mathrm{~mm}$, y su rango menor $0.95 \mathrm{~mm}$ y el mayor $1.45 \mathrm{~mm}$, lo que le hace ser una estructura fina aunque resistente.

\section{BIBLIOGRAFÍA}

3. Aktekin M, Uzmansel DKZ. Examination of the accesory Tendons of Extensor Hallucis Longus Muscle in Fetuses. Clin Anat 2008; 21: 713-717. http://dx.doi.org/10.1002/ca.20712

4. Al-saggaf S. Variations in the insertion of the extensor hallucis longus muscle. Folia Morphol (warsz) 2003; 62: 147-155.

5. Bibbo CAG, Patel DV. The accesory extensor tendon of the first metatarsophalangeal joint. Foot and Ankle 2004; 25: 387-390.

6. Boyd NBH, Meier A, Miller R, Mlady G, Firoozbakhsh K. Extensor hallucis capsularis: frecuency and identification on MRI. Foot and Ankle 2006;27: 181-184.

7. Grant AM, Dalley F. Atlas de Anatomía. Ed Panamericana. Madrid, 2007.

8. Latarjet MRL. Anatomía Humana.3 ${ }^{a}$ Ed Panamericana. Buenos aires, 1997.

9. LLorca O. Anatomía Humana. $5^{\text {a }}$ Ed Científico-médica. Barcelona. 1981.

10. Moore A. Fundamentos de Anatomía con Orientación Clínica. Panamericana. Madrid, 2003.

11. Netter F. Atlas de Anatomía Humana. 4ª Ed Masson. Barcelona. 2007.

12. Olson TR. A.D.A.M. Atlas de Anatomía Humana. Masson. Barcelona. 1997.

13. Platzer. Atlas de Anatomía con correlación clínica. Aparato Locomotor. $9^{a}$ Ed Panamericana. Madrid. 2005.

14. Rohen JW. Atlas de Anatomía Humana. Estudio Fotográfico del Cuerpo Humano. Elsevier. Madrid. 2007.

15. Rouviere HD. Anatomía Humana Descriptiva, Topográfica y Funcional. 11ª Ed Masson. Barcelona. 2005.

16. Sarrafián S. Anatomy of the Foot and Ankle. Descriptive,topographic,functional. $2^{\mathrm{a}}$ Ed L. Company. Philadelphia. 1993.

17. Schünke MSE, Schumacher U. Prometeus. Texto y Atlas de Anatomía. Panamericana. Madrid. 2005.

18. Sobotta. Atlas de Anatomía Humana.Tronco,Visceras y Miembro Inferior.21 ${ }^{\mathrm{a}}$ Ed. Panamericana. Madrid. 2006. 